\title{
Materials evaluation for the Origins Space Telescope
}

\author{
Carly R. Sandin $\odot,{ }^{\mathrm{a}, *}$ Lynn N. Allen, ${ }^{\mathrm{b}}$ Edward G. Amatucci $\odot,{ }^{\mathrm{a}}$ \\ Jonathan W. Arenberg $\odot,{ }^{\mathbf{c}}$ Ruth C. Carter, ${ }^{\text {a James A. Corsetti, }}{ }^{\text {a }}$ \\ Michael J. DiPirro, ${ }^{a}$ Matthew East, ${ }^{\text {b }}$ Louis G. Fantano, ${ }^{a}$ Anel Flores, ${ }^{\text {a }}$ \\ J. Scott Knight, ${ }^{d}$ David T. Leisawitz $\odot,{ }^{a}$ Paul A. Lightsey $\odot,{ }^{d}$ \\ Gregory E. Martins, ${ }^{a}$ Ted Mooney, ${ }^{b}$ Alison Nordt, ${ }^{e}$ and John B. Steeves ${ }^{f}$ \\ ${ }^{a}$ NASA Goddard Space Flight Center, Greenbelt, Maryland, United States \\ ${ }^{b}$ L3Harris Technologies, Rochester, New York, United States \\ ${ }^{\mathrm{c}}$ Northrop-Grumman Space Systems, Redondo Beach, California, United States \\ ${ }^{\mathrm{d} B a l l}$ Aerospace Corporation, Boulder, Colorado, United States \\ e Lockheed-Martin Advanced Technology Center, Palo Alto, California, United States \\ ${ }_{\mathrm{f}}^{\mathrm{f}}$ et Propulsion Laboratory, California Institute of Technology, Pasadena, California, \\ United States
}

\begin{abstract}
The Origins Space Telescope (Origins) study team prepared and submitted a Mission Concept Study Report for the 2020 Decadal Survey in Astrophysics. During the study, a Materials Working Group was formed to evaluate materials for Origins. The Materials Working Group identified material candidates and evaluated the candidates using driving requirements and key material considerations. The evaluation resulted in several options to aid the study team in making a materials selection for the mission concept. Our paper details the approach to the materials evaluation and the results. (c) The Authors. Published by SPIE under a Creative Commons Attribution 4.0 Unported License. Distribution or reproduction of this work in whole or in part requires full attribution of the original publication, including its DOI. [DOI: 10.1117/1.JATIS.7.1.011011]
\end{abstract}

Keywords: materials; cryogenic; mirrors; infrared; space telescope; mission concept.

Paper 20085SS received Jun. 17, 2020; accepted for publication Jan. 12, 2021; published online Feb. 10, 2021.

\section{Introduction}

\subsection{Origins Space Telescope}

The Origins Space Telescope (Origins) traces our cosmic history, from the formation of the first galaxies and the rise of metals to the development of habitable worlds and present-day life. Origins does this through exquisite sensitivity to infrared radiation from ions, atoms, molecules, dust, water vapor, and ice, and observations of extra-solar planetary atmospheres, protoplanetary disks, and large-area extragalactic fields. Origins operates in the wavelength range 2.8 to $588 \mu \mathrm{m}$ and is more than 1000 times more sensitive than its predecessors due to its large, cold $(4.5 \mathrm{~K})$ telescope and advanced instruments. ${ }^{1}$

\subsection{Materials Evaluation}

A materials evaluation was completed for the mission's main optical and structural elements. The evaluation team, which included Origins team members and industry partner materials experts, developed an iterative process to identify suitable material candidates. The material candidates under consideration were chosen based on their material properties, spaceflight mission heritage, and knowledge of current manufacturing and processing capabilities. The evaluation criteria were determined by the driving requirements for Origins, material performanceparticularly at cryogenic temperatures - and relevant manufacturing challenges.

An initial assessment of primary mirror material candidates yielded five potential options: beryllium, aluminum, AlBeMet ${ }^{\circledR}$, silicon carbide (SiC), and fused silica. The team conducted

*Address all correspondence to Carly Sandin, carly.sandin@nasa.gov 
further trade studies to better understand the material performance needed to meet Origins' requirements. Structural materials were also evaluated in a broader framework. This paper describes the approach to the evaluations and the results, ultimately leading to a materials selection.

\section{Evaluation Criteria}

\subsection{Driving Requirements}

The telescope, including the backplane, is expected to be isothermal so it must be composed of materials with relatively high thermal conductivity, greater than $4 \mathrm{~W} / \mathrm{m} \cdot \mathrm{K}$ at $4.5 \mathrm{~K}$. It must have low overall mass, namely $<900 \mathrm{~kg}$, to be consistent with the $6643 \mathrm{~kg}$ payload mass established for the design, ${ }^{1}$ a large primary mirror with a light collecting area $>25 \mathrm{~m}^{2}$, and the materials themselves should have a relatively high technology readiness level (TRL) $\geq 4$, where component validation exists in a laboratory environment.

\subsection{Evaluation Criteria}

The evaluation criteria include key material properties that drive performance for the Origins' primary mirror and additional considerations. The criteria include the following: density, stiffness, thermal conductivity, coefficient of thermal expansion (CTE), outgassing, and manufacturability.

\subsubsection{Density}

Telescope mass is driven by two factors: size and material density. The size is driven by the science objectives, including the aperture diameter of the primary mirror and number and types of instruments. Density is one of the most significant material properties for systems launching to space. Materials with a low density and high overall material performance are ideal for meeting Origins' overall mass budget.

\subsubsection{Stiffness}

Stiffness, or Young's modulus, is an especially important material property for optical elements and some structural elements. High stiffness provides dimensional stability, allowing mirrors and structural components to hold their shape over long periods. Stiffness is also critical for maintaining optical alignments in the system, especially during launch and deployment when a telescope experiences severe vibrations. ${ }^{2}$ Specific stiffness, or specific modulus, is Young's modulus per mass density. Materials with high specific stiffness are generally favored because components have maximum stiffness at minimum weight, an attractive goal for Origins when considering the mass budget, elements, and gravity impacts while verifying the 0 -g optical system in $1 \mathrm{~g}$.

\subsubsection{Thermal conductivity}

A unique objective of Origins is achieving a temperature of $4.5 \mathrm{~K}$ on the cold side of the telescope. Materials with high thermal conductivity can transfer heat faster and cool down uniformly. The primary mirror is one example of where thermal conductivity is critical. Cryocooler heat exchangers are strategically placed on the back of the Origins primary mirror structure and need to cool down the entire mirror, including mirror segments further away from the cryocoolers. The connecting elements of the mirror-segment frames and struts-should also have a reasonable thermal conductivity to enable them to reach $4.5 \mathrm{~K}$ and be isothermal under reasonable $(\sim 10 \mathrm{~mW})$ heat flows at operating temperature. It is estimated that it will take only a few days for the telescope and instruments to reach operating temperature. ${ }^{1}$ On the other hand, the warm areas of Origins need to be isolated from the cold components to prevent heat transfer. Therefore, some sections of the telescope need to be made from materials with very low thermal conductivity. 


\subsubsection{Coefficient of thermal expansion}

The CTE is used to determine how a material changes dimensionally as a function of temperature. It is a critical property for structures comprised of different materials, especially for bonds and joints that use polymers or metal nodes. Using materials with similar CTEs can reduce or eliminate issues with thermomechanical stresses at interfaces or in bonding materials. Some materials, such as Invar, have an extremely low CTE and are typically used as fittings to mitigate CTE mismatch between structural components, such as composites tubes, rather than using polymers or metals with large thermal expansions. It is important to consider the CTE over the entire temperature range, from room temperature to $4.5 \mathrm{~K}$, to account for dimensional changes during cool down. It is also crucial to account for the CTE at $4.5 \mathrm{~K}$, to design a stable and functioning system at operating temperature. A related property, thermal strain, is the strain in the material caused by the temperature change, from room temperature to $4.5 \mathrm{~K}$ for Origins. The CTE at $4 \mathrm{~K}$ and the thermal strain from 293 to $4 \mathrm{~K}$ are plotted against specific stiffness for five potential materials in Sec. 3.3. Steady-state gradient thermal stability (thermal conductivity/CTE) and long-term stability (thermal diffusivity/CTE) are two other significant thermal considerations that compound with CTE at $4 \mathrm{~K}$ and drive long-term operating stability and gradient stability. Both are also plotted in Sec. 3.3.

\subsubsection{Outgassing}

It is crucial for the optics to remain clean before launch and in space so they can provide clear images and high-quality data during operation. Some materials may outgas in a vacuum environment depending on their material composition and temperatures. Outgassing is a concern because the released gasses, including water vapor, can potentially condense on cold optical surfaces and distort images and data. During deployment, materials may outgas when in direct view of the sun. The outgassing properties of all organic materials need further evaluation, including adhesives and resin matrix materials in composites such as carbon fiber reinforced polymer (CFRP), polyether ether ketone (PEEK), Ultem ${ }^{\circledR}$, and Vespel ${ }^{\circledR}$.

\subsubsection{Manufacturability}

When selecting a primary mirror, whether it is a monolithic mirror or a segmented mirror, the material choice can have significant impacts on development. For example, a monolithic mirror made of a material with limited heritage may require an extensive facility development program to accommodate the size and material selected whereas a material with heritage may allow for the use of existing equipment. A segmented mirror may instead require a lengthy segment development program, where the first segment is manufactured and tested and the remaining segments are manufactured subsequently. James Webb Space Telescope (JWST) underwent an extensive segment development program for the 18 beryllium segments for its $6.5-\mathrm{m}$ primary mirror. ${ }^{3}$

The team considered heritage and meter-class mirror manufacturability for point to point hexagonal segments for all material candidates. Cryogenic testing is also a lengthy process for mirror development. However, Origins' relaxed requirements in comparison to JWST-Origins is diffraction limited at $30 \mu \mathrm{m}$ compared to JWST at $2 \mu \mathrm{m}$-may eliminate the need for some testing, such as cryo-null testing and figuring for mirror shape, which helps reduce the cost and schedule for mirror development.

\section{Mirror Materials}

\subsection{Preliminary Assessment}

The mirror material evaluation was prioritized over the structural material evaluation due to the inherent complexity of assessing mirror manufacturing. A preliminary list of material candidates is shown in Table 1. Candidate mirror materials were systematically evaluated, resulting in five potential options. Major advantages and disadvantages were noted for each material with respect to manufacturability and material performance for Origins. The candidates were selected due to

J. Astron. Telesc. Instrum. Syst. $\quad$ 011011-3 Jan-Mar 2021 • Vol. 7(1) 
Table 1 Candidate mirror materials were systematically evaluated, resulting in five potential options.

\begin{tabular}{|c|c|c|}
\hline Material & Advantages & Disadvantages \\
\hline \multicolumn{3}{|l|}{ Potential materials } \\
\hline Beryllium 0-30 & $\begin{array}{l}\text { Superior stiffness, extremely lightweight, } \\
\text { and low CTE over temperature range }\end{array}$ & $\begin{array}{l}\text { Expensive, brittle, toxic, and long } \\
\text { machining time }\end{array}$ \\
\hline Aluminum 6061 & $\begin{array}{l}\text { Good structurally, good fabrication time, } \\
\text { and inexpensive }\end{array}$ & Heavy, reactive surface, and low stiffness \\
\hline AlBeMet $^{\circledR}$ & $\begin{array}{l}\text { Good stiffness, lightweight, and low CTE } \\
\text { over temperature range }\end{array}$ & Limited information and heritage, toxic \\
\hline $\mathrm{SiC}$ & $\begin{array}{l}\text { Excellent stiffness, excellent strength, } \\
\text { and low CTE over temperature range }\end{array}$ & $\begin{array}{l}\text { Brittle, heavy, expensive, and long } \\
\text { machining time }\end{array}$ \\
\hline Fused Silica & $\begin{array}{l}\text { Low CTE over temperature ranges and } \\
\text { lightweight }\end{array}$ & Low stiffness and low thermal conductivity \\
\hline \multicolumn{3}{|c|}{ Eliminated materials } \\
\hline Titanium & $\begin{array}{l}\text { Excellent strength and good thermal } \\
\text { performance }\end{array}$ & Extremely heavy and low machinability \\
\hline ULE & Low CTE and lightweight & Poor thermal performance and low strength \\
\hline Zerodur & Low CTE & Poor thermal performance and low strength \\
\hline Borosilicate & Lightweight & Poor thermal performance and low strength \\
\hline Composite/CFRP & $\begin{array}{l}\text { Extremely lightweight and low CTE can } \\
\text { be achieved }\end{array}$ & $\begin{array}{l}\text { Limited manufacturability technology and } \\
\text { creep }\end{array}$ \\
\hline
\end{tabular}

their material properties, mirror material heritage, and the knowledge of current manufacturing capabilities.

Traditional mirror materials include glasses, ceramic materials, and fused quartz while nontraditional mirror materials include metals, metal alloys, SiC, and CFRP composites. Nontraditional mirror materials offer opportunities to reduce weight and cost. In the case of Origins, nontraditional mirror materials also provide thermal advantages over traditional mirror materials. ${ }^{4}$ The first assessment was primarily driven by Origins' $4.5 \mathrm{~K}$ operating temperature. The first materials eliminated were any that were not ideally suited for cryogenic temperatures. This included the glasses and glass ceramics: Ultra Low Expansion (ULE) glass (titania-silicate glass), Zerodur (lithium-aluminosilicate glass-ceramic), and Borosilicate (glass with silica and boron trioxide). However, fused silica was not eliminated because of its prominent heritage as an optical substrate and its potential to perform in cryogenic temperatures. Other materials eliminated include titanium because of its extremely high density and CFRP based on its limited manufacturability technologies. Five mirror material candidates remained: beryllium, aluminum, fused silica, $\mathrm{SiC}$, and aluminum, and beryllium metal matrix composite (AlBeMet ${ }^{\circledR}$ ).

\subsubsection{Fused silica}

Fused silica has extensive heritage as an optical substrate. It also has the lowest CTE at $4.5 \mathrm{~K}$, nearly indistinguishable from beryllium, the lowest strain at $4.5 \mathrm{~K}$ and relatively low specific stiffness. However, it has poor thermal conductivity and thermal diffusivity, which is critical for Origins. Thermal diffusivity is the thermal conductivity divided by density and specific heat capacity at constant pressure, measuring the rate of heat transfer in a material. In terms of manufacturability, it is roughly equivalent to ULE glass with boule production, light-weighting, and polishing. 


\subsubsection{Silicon carbide}

Silicon carbide $(\mathrm{SiC})$ has high specific stiffness, low strain at $4.5 \mathrm{~K}$-significantly higher than fused silica but lower than others-low CTE at $4.5 \mathrm{~K}$, and excellent thermal conductivity and diffusivity. It also has the potential for many segments to be created from a single mold using a cladding process. SiC has spaceflight heritage through the Herschel Space Observatory, a large 3.5-m sintered $\mathrm{SiC}$ primary mirror. ${ }^{5}$ Other examples of $\mathrm{SiC}$ heritage include a $\mathrm{SiC}$ bench for near-infrared spectrograph Instrument on JWST and SiC mirrors have been demonstrated on GAIA of the European Space Agency (ESA). ${ }^{6,7}$

\subsubsection{Beryllium}

Beryllium has the highest performance because of its high specific stiffness, low CTE at $4.5 \mathrm{~K}$, and excellent thermal conductivity and diffusivity. It also has the most relevant heritage through the JWST with a large, segmented primary mirror operating at cryogenic temperatures. It also requires significant schedule lead time to develop segments and imposes higher costs, mainly associated with manufacturing. Even though beryllium is high TRL based on its JWST heritage, it requires extra care in manufacturing due to the human health complexity factor in grinding and light-weighting.

\subsubsection{Aluminum 6061}

Aluminum 6061 has good thermal conductivity and diffusivity. It is also excellent for manufacturability because it can be machined easily, polished, and heat-treated. However, its high density, extremely high strain at $4.5 \mathrm{~K}$-highest of all the materials-and relatively high CTE at $4.5 \mathrm{~K}$ make it an overall poor performer for Origins. An athermal design, both optically and structurally, would minimize some issues associated with strain and CTE mismatch, but then mass is an issue. Options exist to improve light-weighting for an aluminum mirror but there is also chemical stability to consider because of its highly reactive surface. Spaceflight heritage exists for apertures below $0.5 \mathrm{~m}$.

\subsubsection{AlBeMet ${ }^{\circledR}$}

AlBeMet ${ }^{\circledR}$ is a metal matrix composite of aluminum and beryllium. Its materials properties fall between beryllium and aluminum and while it has a better performance than aluminum, it still has some of the disadvantages of low manufacturability due the toxicity of beryllium while also being lower TRL. It also lacks heritage, with little-to-no spaceflight heritage and no meter class heritage. ${ }^{8}$

\subsection{Mirror Material Trade Matrix}

A material trade matrix was created for the top choices among the remaining material candidates. This trade matrix was initially designed for consideration of passive mirror segments for a $9.1-\mathrm{m}$ aperture, where the actuators are used once to optically phase the mirror segments. The matrix is shown in Table 2 and assesses each material on a scale of 1 to 5 for performance, schedule, and cost, using NASA standard values. The materials are listed in order of preference, showing fused silica and $\mathrm{SiC}$ as the top choices.

Additionally, the results of the mirror material trade matrix show beryllium as the highest performing, despite programmatic challenges. $\mathrm{SiC}$ also has high performance and is another viable option for Origins. AlBeMet ${ }^{\circledR}$ offers the opportunity to improve the manufacturability of beryllium but would ultimately weigh more overall and have a lower stiffness than beryllium. Fused silica has the strongest optical heritage among the candidates, which would greatly reduce cost and schedule, but its lower thermal conductivity makes it less ideal for cooling than the other candidates. Aluminum has relatively good properties and machinability, but overall poorer thermal contraction performance and lower specific stiffness than the top candidates. 
Table 2 A material trade matrix assisted the Origins team in identifying the top primary mirror materials. (On a scale of 1 to 5 with the higher values being better.)

\begin{tabular}{lcccl}
\hline \hline Material & Performance & Schedule & Cost & \multicolumn{1}{c}{ Heritage } \\
\hline Fused Silica & 3 & 5 & 4 & Glass has max heritage as optic substrate \\
SiC (multiple) & 4 & 4 & 3 & Herschel heritage \\
Beryllium & 5 & 2 & 2 & JWST heritage \\
Aluminum 6061 & 2 & 5 & 5 & All-Al telescope studied, found to be too massive \\
AlBeMet & & 2 & 1 & No meter-class heritage \\
\hline \hline
\end{tabular}

\subsection{Material Performance for Origins}

The team created material performance plots to further evaluate the five potential material candidates. Figure 1 shows critical material properties plotted against specific stiffness at room temperature for beryllium, $\mathrm{AlBeMet}^{\circledR}, \mathrm{SiC}$, fused silica, and aluminum. It is important to note that measured material properties vary at $4.5 \mathrm{~K}$ from test to test and alloy to alloy. The properties also vary based on purity, grade, and manufacturer. Where $4 \mathrm{~K}$ data is unavailable, values measured at temperatures in the range 4.5 to $30 \mathrm{~K}$ were used in these figures.

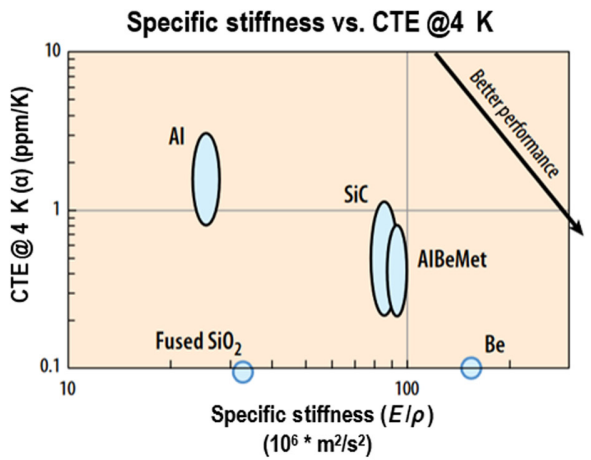

(a)

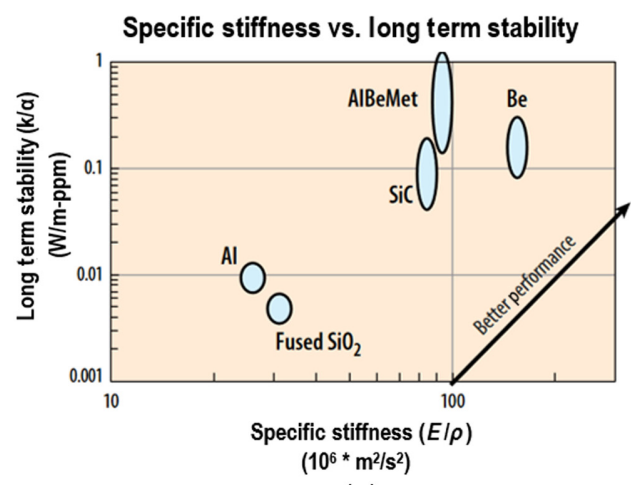

(c)

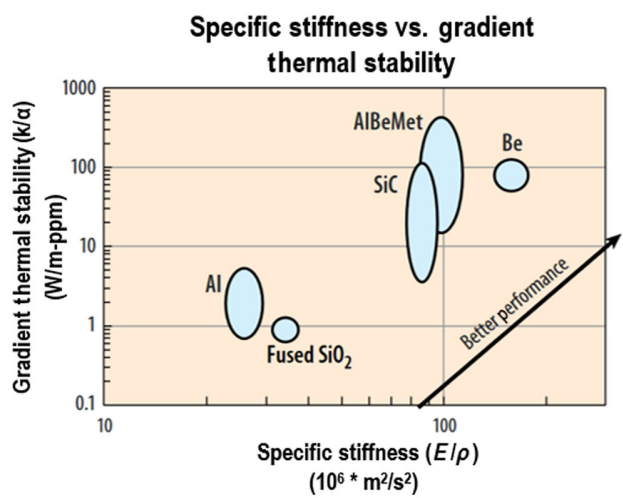

(b)

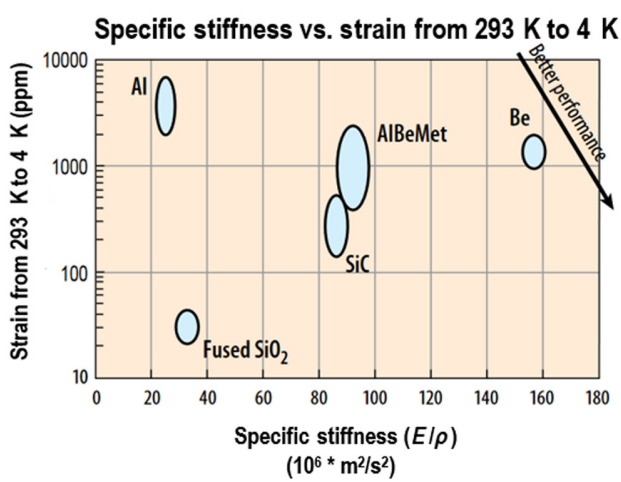

(d)

Fig. 1 (a) The CTE at $4 \mathrm{~K}$ plotted against specific stiffness shows beryllium (Be) is the best performer, followed by AIBeMet ${ }^{\circledR}, \mathrm{SiC}$, fused silica, and aluminum (AI). (b) Steady-state gradient thermal stability plotted against specific stiffness shows Be, AlBeMet ${ }^{\circledR}$, and $\mathrm{SiC}$ as the best performers. (c) Long-term stability plotted against specific stiffness shows Be, AlBeMet ${ }^{\circledR}$, and $\mathrm{SiC}$ as the best performers. (d) Strain from room temperature $(293 \mathrm{~K})$ down to $4 \mathrm{~K}$ against specific stiffness shows trade-offs between $\mathrm{Be}, \mathrm{AlBeMet}{ }^{\circledR}, \mathrm{SiC}$, and fused silica, with $\mathrm{Al}$ as the lowest performer. 
Table 3 The advantages and disadvantages of each structural material were considered.

\begin{tabular}{|c|c|c|}
\hline Material & Advantages & Disadvantages \\
\hline Aluminum & $\begin{array}{l}\text { Good fabrication time, inexpensive, and } \\
\text { high thermal conductance }\end{array}$ & $\begin{array}{l}\text { Heavy in comparison to CFRP and low } \\
\text { stiffness }\end{array}$ \\
\hline Copper & Excellent thermal properties & Extremely heavy \\
\hline Composite/CFRP & $\begin{array}{l}\text { Extremely lightweight and low CTE can } \\
\text { be achieved }\end{array}$ & Creep and outgassing \\
\hline Titanium & $\begin{array}{l}\text { Excellent strength and good thermal } \\
\text { properties }\end{array}$ & Extremely heavy and machining ability \\
\hline Invar & $\begin{array}{l}\text { Near zero CTE, excellent stiffness, and } \\
\text { strength }\end{array}$ & Heavy \\
\hline Stainless steel & Excellent structural material & Extremely heavy \\
\hline Beryllium & $\begin{array}{l}\text { Superior stiffness, extremely lightweight, } \\
\text { low CTE, and high thermal conductance }\end{array}$ & $\begin{array}{l}\text { Expensive, brittle, toxic, and long } \\
\text { machining time }\end{array}$ \\
\hline $\mathrm{SiC}$ & $\begin{array}{l}\text { Excellent stiffness, excellent strength, } \\
\text { and low CTE }\end{array}$ & Brittle, expensive, and long machining time \\
\hline
\end{tabular}

\section{Structural Material}

Ideal structural materials share many of the same characteristics as ideal mirror materialsstrong, lightweight, and a near-zero change in thermal expansion. The team considered aluminum, copper, CFRP, titanium, Invar, stainless steel, beryllium, and SiC as the possible structural materials to use on Origins. Table 3 summarizes the overall appraisal.

Standard metals are typically strong and have excellent thermal properties but are generally too heavy to be used in large components. Lightweight materials, such as beryllium and SiC, are ideal but require more design development for large structural components. CFRP is strong, lightweight, but has low thermal conductance and is not suitable for Origins without also providing extensive thermal strapping to isothermalize the structure. Its other weakness is that it will produce water outgassing when warm $(>160 \mathrm{~K})$.

\subsection{Final Materials Selection}

For the $4.5 \mathrm{~K}$ structure, beryllium was selected. There are several potential issues with the use of beryllium that will be taken into account. Beryllium is more brittle compared to traditional materials such as aluminum so it requires special care in design (sharp corners, proper clearance holes, etc.). Beryllium dust is toxic, so fabrication is limited to certain places. These dangers do not exist after fabrication though, and a safety and handling plan would be created to address transport and handling of the parts.

Several organizations that have used large beryllium structures were consulted and offered guidance for Origins. These organizations would not hesitate to use beryllium again. Ball Aerospace developed the beryllium mirrors and the beryllium Aft Optics Subsystem (AOS) bench for the $\mathrm{JWST}^{9}$ as well as the beryllium mirror and structure for Spitzer. ${ }^{10,11}$ Lockheed $^{2}$ Martin developed the beryllium bench of near-infrared camera (NIRCam) on JWST. ${ }^{2}$ Origins plans to work with vendors early to qualify workmanship and design details. A segment of the primary mirror would be used to validate performance and then again after integrating the segments to the mirror backplane. Origins recognizes the need to restore the beryllium processing facilities used by JWST.

For the spacecraft structure, the team selected CFRP whenever possible. Origins has critical structures on the warm side [spacecraft bus module (SBM)] and the cold side (mirror and instrument thermally isolating support structures). While the observatory is warm before cool down, water vapor will outgas from CFRP. On the cold side, this vapor could condense onto the mirror

J. Astron. Telesc. Instrum. Syst. $\quad$ 011011-7 Jan-Mar 2021 • Vol. 7(1) 
and instruments, impacting optical throughput. As a result, the team avoided using CFRP as much as practicable on the cold side, opting instead for metals.

Therefore, CFRP is an advantageous choice for the SBM, whereas metals are more suited for the cold side structures. An exception to this are the $4.5 \mathrm{~K}$ bipods, which are made of the CFRP M55J to provide the necessary stiffness to thermal conductivity in the range of 4.5 to $35 \mathrm{~K}$. In contrast, high thermal conductance is critical for the backplane, which will be cooled conductively to $4.5 \mathrm{~K}$, so the team sought a metal with good thermal conductivity down to $4.5 \mathrm{~K}$. The mirror structures would also ideally be made from the same material as the mirror segments to avoid CTE mismatch between structures on the cold side. Thus, an athermal design of beryllium on the cold side is favorable.

\section{Conclusion}

The materials selection is a trade-off process. The most important parameters for the Origins primary mirror are specific stiffness (high stiffness and low density) and thermal performance (high thermal conductivity and low CTE). Beryllium O-30 has excellent specific stiffness and adequate thermal performance for Origins' needs. It is also the highest TRL material and has the most relevant cryogenic heritage, which advances the manufacturability and development. SiC is an attractive alternative option, but requires trades on the design and processing. Further assessments to address face sheet thickness, rib thickness, cell size, and mounting configuration of the mirror segments are recommended for either material option.

For structural materials, an athermal design is an attractive solution for CTE matching but greatly increases the mass. As a result, the team recommends composites whenever possible. For the backplane and other cold side structures, thermal performance is more important than mass. Therefore, the team recommends these structures be made of the same material as the primary mirror. However, beryllium and $\mathrm{SiC}$ require more design development in comparison to most metals and composites, so early work with potential designs and vendors is needed to ensure larger structures made of these materials will meet performance requirements and survive launch.

\section{Acknowledgments}

This work summarizes the results of the materials evaluation as reported in the Origins Space Telescope Mission Concept Study Final Report. This work would not have been possible without the industry partners and Origins team members who supported and participated in the Origins Materials Working Group. We would like to thank all the institutions that contributed and to the National Aeronautics and Space Administration (NASA) for providing support and funding.

\section{References}

1. "Origins Space Telescope mission concept study report," NASA (2019).

2. D. J. Edinger and A. A. Nordt, "Selection of I-220H beryllium for NIRCam optical bench," Proc. SPIE 5868, 58680X (2005).

3. "Mirrors Webb/NASA," NASA, www.jwst.nasa.gov/content/observatory/ote/mirrors/index .html.

4. J. Cheng, "Mirror design for optical telescopes," in The Principles of Astronomical Telescope Design, Astrophysics and Space Science Library, pp. 377-441, Springer, New York (2009).

5. G. L. Pilbratt, "Herschel mission overview and key programmes," Proc. SPIE 7010, 701002 (2008).

6. M. Bougoin and J. Lavenac, "Large and highly stable structures made of SiC," in Proc. 12th Eur. Conf. Spacecraft Struct., Mater. and Environ. Testing, ESA SP-691, p. 49 (2012).

7. M. te Plate et al., "The European optical contribution to the James Webb Space Telescope," Adv. Opt. Technol. 7(6), 353-364 (2018).

8. M. East et al., "Material selection for far infrared telescope mirrors," Proc. SPIE 10698, $106981 \mathrm{~N}$ (2018). 
9. K. Martinez et al., "Cryogenic design and predicted performance of the James Webb space telescope beryllium aft optics subsystem optical bench," Proc. SPIE 6666, 66660S (2007).

10. “The Telescope," NASA Spitzer Space Telescope, NASA's Jet Propulsion Laboratory, www.spitzer.caltech.edu/mission/180-The-Telescope.

11. D. B. Gallagher, W. R. Irace, and M. W. Werner, "The development and mission of the Space Infrared Telescope Facility (SIRTF)," Proc. SPIE 5487, 13-25 (2004).

Carly R. Sandin is a materials and processes engineer at NASA Goddard Space Flight Center. She supports the Nancy Grace Roman Space Telescope and previously supported the Robotic Refueling Mission 3. She received her MS degree in mechanical engineering from the University of Illinois Urbana-Champaign and her BS degree in mechanical engineering from South Dakota School of Mines and Technology.

Edward G. Amatucci has developed everything from robotic devices to scientific instruments for space missions over the past 38 years as an engineer, and currently is an instrument systems engineer as a contractor for NASA Goddard Space Flight Center. He earned his bachelor of science degree in mechanical engineering from the University of Maryland, a master of engineering in mechanical engineering, and master of science in technical management from Johns Hopkins University.

Jonathan W. Arenberg is the chief engineer for Space Science Missions at Northrop Grumman Space Systems. He has worked on the Chandra X-ray Observatory, the Starshade, James Webb Space Telescope, technology development, and numerous mission studies, including Origins. His work experience also includes high energy lasers and other optical technologies. He is the author of over 200 conference presentations, papers, a book and book chapters, holds 13 patents and is an SPIE Fellow.

Ruth C. Carter was a systems engineer and space flight mission development manager at the NASA Goddard Space Flight Center from 1986 to 2019 (retired, 2019). She managed and led a team to design and develop flight systems, including Hubble Space Telescope Servicing Missions, Tropical Rainfall Measuring Mission, and the Origins Space Telescope mission study. She received her BS and MS degrees in nuclear engineering from the University of Maryland at College Park.

Michael J. DiPirro received his $\mathrm{PhD}$ in low-temperature physics from the State University of New York at Buffalo, and a one-year National Research Council Postdoctoral Fellowship at the National Institute of Standards and Technology. Then, he joined NASA Goddard in 1980. $\mathrm{He}$ has worked on a number of astrophysics missions over the last 40 years, including the Cosmic Background Explorer (COBE), Astro-E, -E2, and -H, XRISM, Spitzer, the Wide-field Infrared Explorer (WIRE), the Wide-field Infrared Survey Explorer (WISE), and JWST. Between COBE and Astro-E he was the principal investigator on the Superfluid Helium On-Orbit Transfer Flight Demonstration, and Co-I on a Cross Enterprise Technology Development Program to develop a new type of adiabatic demagnetization refrigerator. $\mathrm{He}$ is currently the technical lead and chief technologist for the Origins Space Telescope study for the 2020 Astrophysics Decadal Survey.

Matthew East innovates large optics as a lead opto-mechanical engineer at L3HarrisTechnologies in Rochester, New York. Since age one, he regularly attended telescope making conventions, learning about optical systems, fabrication, and metrology. He has patented inventions in additive manufactured optics and has published concepts for spaceborne astronomy missions. He holds a BSME from Rensselaer Polytechnic Institute and an MS in engineering management from Clarkson University. His goal is to drive the next generation of technology that enables breakthrough astronomical observations.

Louis G. Fantano received his BS degree in chemical engineering from the University of Notre Dame in South Bend, Indiana, and joined Rockwell International as a thermal engineer on the Space Shuttle program in 1981. He began at NASA in 1991 and served as the lead thermal engineer for numerous flight programs, including the Landsat-8, Glory, Magnetospheric

J. Astron. Telesc. Instrum. Syst. $\quad 011011-9 \quad$ Jan-Mar 2021 • Vol. 7(1) 
Multiscale Mission, Fermi Gamma-ray Space Telescope, Sub-millimeter Wave Astronomy Satellite, and Geostationary Operational Environmental Satellites flight projects.

J. Scott Knight is a senior staff consultant in Missions Systems Engineering at Ball Aerospace. His current focus is payload architecture for large space systems. He has been involved with segmented space telescopes for over 15+ years, concentrating on system engineering, modeling, test design for ground-based verification and flight commissioning.

David T. Leisawitz is NASA study scientist for the Origins Space Telescope and is most interested scientifically in the development of habitable conditions during planet formation. He was PI on the Space Infrared Interferometric Telescope mission concept study and served as NASA Goddard study lead for the Submillimeter Probe of the Evolution of Cosmic Structure. He was mission scientist for the Wide-field Infrared Survey Explorer and deputy project scientist for the COBE.

Paul A. Lightsey has 50+ years'experience including work on HST instruments, Webb, and studies for future large space telescopes. He has coauthored a book on systems engineering of astronomical telescopes. He received his BS degree from Colorado State University, a PhD from Cornell University, and received his Distinguished Public Service Medal from NASA. He is a SPIE Fellow and a senior member of OSA.

Biographies of the other authors are not available. 